\title{
mHealth - areas of application and the effectiveness of interventions
}

\author{
Mariusz Duplaga $\odot$ htpps///orid.orgy0000-0001-6963-8414 \\ Anna Tubek (ํ) htps://orid.ory/0000-0001-966-2125
}

Department of Health Promotion Institute of Public Health Faculty of Health Sciences Jagiellonian University Collegium Medicum, Krakow, Poland

Address for correspondence: Mariusz Duplaga, Instytut Zdrowia Publicznego, Grzegórzecka 20, 31-531 Kraków, mariusz.duplaga@uj.edu.pl

\section{Abstract}

mHealth is seen as the next stage of developing applications for information and communication technologies in the area of health care. This paper presents the evolution of mHealth systems and the results of the latest analyses of the effectiveness of mHealth interventions. Intensive mHealth development results from the near universal access to mobile devices, primarily smartphones, and devices that use sensory technologies. The great interest in mobile applications is reflected in the offer of online stores, which already provide many thousands of programs that can be installed on a mobile phone. Of the many possible mHealth applications, those supporting chronic diseases care and health promotion activities are regarded as the most promising. The growing interest in the area of mHealth is proven by the large number of publications that present syntheses of the available evidence. Although studies aimed at assessing the usefulness and effectiveness of health applications quite often raise doubts about the methodological quality, the significance of the analyses is positive. However, problems in maintaining the involvement of health users in mHealth solutions may be a cause for concern.

\section{Key words: mHealth, health interventions, information and communication technologies}

Stowa kluczowe: m-zdrowie, interwencje zurowotne, technologie informacyjne i komunikacyjne

Ministerstwo Nauki i Szkolnictwa Wyższego
Przygotowanie do wydania elektronicznego finansowane w ramach umowy 641/P-DUN/2018 ze środków Ministra Nauki i Szkolnictwa Wyższego przeznaczonych na działalność upowszechniającą naukę.

\section{Introduction}

mHealth technologies are often regarded as the most innovative developments in the areas in health care and public health. Most authors agree that they provide great opportunities for enhancing health promotion, disease prevention and medical care. This seems to be confirmed by the study undertaken by Mechael and Slonisky under the auspices of the World Health Organization (WHO) and updated as part of the Millenium Villages Project in 2008 [1]. The general access to multi-functional mobile devices makes them an obvious choice for supporting the health of individuals in their daily lives.
The potential, resulting from the use of telecommunications and information technologies in health care, has been noticed since the first half of the $20^{\text {th }}$ century. Admittedly, the technologies available at the time did not allow for the implementation of practical solutions, but the vision of the implementation of medical services "at a distance" was drawn in the imagination of people watching the technical developments. This is evidenced by the notion of a "telemedical station" shown on the cover of the journal Radio News in 1924 [2] or a device called a "teledactyl", described by Gernsback in the Science Invention journal in 1925 [3]. The more complex telemedicine systems first began to appear only in the 
1950s, thanks to the use of television, which rapidly grew in popularity and importance [4]. Paradoxically, the first definitions of telemedicine, stating that it is a form of providing medical services without direct contact by the participants, but using audiovisual transmission came as late as the 1970s [5-7].

Progress in telecommunications and the development of the Internet have led to a situation in which access to elaborate telemedicine applications has become easier and more widespread. The potential of online technologies was soon noticed as being a method for the improvement of medical care and for the implementation of public health goals and health promotion. As early as 1999 , using the analogy of e-commerce sector, the term eHealth was formulated, primarily meaning the provision of medical services via the Internet $[8,9]$. However, as early as 2001, Eysenbach pointed to the significant relationship between the development of the eHealth area and public health. He defined eHealth as "an emerging field in the intersection of medical informatics, public health and business, referring to health services and information delivered and enhanced through the Internet and related technologies" [10]. However, many definitions of eHealth were formulated subsequently. Soon the concept gained much popularity and was used to determine a whole range of applications of information and communication technologies related to health and medical care. It seems that such an understanding of eHealth is now quite common, which has been confirmed by the definition used by WHO [11]. However, it is worth remembering that some authors attribute a broad meaning to telemedicine [12], considering e-health as an area of business operations related to health. In this paper, the meaning of eHealth as confirmed by WHO is retained, whereas the concept of telemedicine, followed by many other authors, will be used for the solutions having a clinical context.

Some authors treat eHealth as being a subsequent stage to telemedicine in the development of telecommunications and information technologies in the sphere of health and medicine. Following this trend, it can be said that the next stage of evolution after eHealth is mHealth. This perception of mHealth may be misleading because this concept appeared almost in parallel with eHealth. As early as 2000, Laxminarayan and Istepanian used the term "unwired e-med" with reference to the next generation of "wireless and Internet telemedicine systems" [13]. It is worth remembering that, despite the first attempts to create a wireless telephony network in the 1950s, the first commercial systems were available only at the beginning of the 1980s. The first fully automatic first generation cellular telephone system was launched in 1981 in Scandinavia with the second generation introduced in Finland in 1991. The first smartphone is considered to be the Nokia Communicator device from 1996, which enabled access to the Internet and the wireless sending of e-mails [14]. It should also be remembered that the set of standards referred to as Wi-Fi (from wireless fidelity), used to build wireless computer networks, appeared on the market in 1999. The widespread development of local networks us- ing radio communication is related to the development of the IEEE 802.11 standard used in products marked with the Wi-Fi trademark [14]. Thus, it can be concluded that the dynamic expansion of the Internet has overlapped the development of wireless communication and has led to the development of new applications such as mHealth. Laxminarayan and Istepanian soon appreciated that wireless connectivity, with the ability to send large volumes of data, could shape a new model of medical care based on the increased participation of citizens and patients [13]. Among the basic benefits resulting from the implementation of such a model of care, they pointed out the speed of emergency response, the flexible and quick access to specialist advice at the place of care, the support for interactive medical consultations, the possibility of sending medical images and video recordings, the management of medical services in areas suffering from a lack of access to medical care and finally the improvement of care in emergency medicine, especially in the case of natural and man-made disasters [13]. In 2003, Istepanian and Lacal defined mHealth as "the use of telecommunications and multimedia technologies in mobile and wireless systems for the provision of health services" [15]. A year later, Istepanian also stated that mHealth means the use of "mobile data processing, medical sensors and communication technologies in health care" [16]. The review published under the auspices of WHO in 2008 indicated that the development of mHealth systems required the crucial use of the opportunities offered by the new generations of mobile communication (GPRS, 3G and 4G systems) as well as of the global positioning system (GPS) and Bluetooth technology [1]. Many authors perceive mHealth as a great opportunity to improve health services. These expectations result from the attributes of mHealth systems, such as interactivity, multi-directional communication, personalisation, the lack of time constraints, sensitivity to the context and its availability and ubiquity [17]. The development of mHealth systems is fostered by the trends observed in modern health care systems, including the emphasis on evidence-based strategies, personalisation of health interventions, development of coordinated or shared care as well as the independence and activation of patients.

\section{The evolution of mHealth solutions}

The history of mobile devices dates back to the $14^{\text {th }}$ century. It is believed that the first watch worn by a user originated in 1511 [18]. Undoubtedly, there was a very long wait for further progress in this area [19]. In 1972, the first digital watch with a liquid crystal display (LED) was produced by Hamilton/Pulsar. Multifunctional devices worn on the wrist, but without access to wide area networks were popular in the 1990s. In the early 2000s, multifunctional devices providing access to the network became available. Since then, advances in the wearable technologies, also known in Poland as "nosidła" [20] have been very rapid. The progress has been associated with the development of sensory technologies which are able to measure various physiological parameters $[19,21]$. 
Another significant breakthrough in the development of mobile applications was the introduction of devices using the $3 \mathrm{G}$ and $4 \mathrm{G}$ mobile networks for data transmission [14]. mHealth has been included in the vanguard of applications using the new opportunities offered by telecommunication. Consequently, the requirement for having access to health and medical services "everywhere and at any time" could be fully implemented.

The smartphone has become the symbol of mobile technologies used for health care. Its popularity arises from it being a portable device providing constant access to the Internet, as well as having the computing power to manage the complex applications needed by doctors and patients. In addition, it is a multifunctional device being used for various purposes and is usually in the user's possession throughout the day. It is also important to realise that there are ever more medical mobile devices on the market which can cooperate with smartphones, e.g. sensors for measuring glycemia, or for measuring physical activity. In most cases, users do not part with their smartphones and, therefore, they can be used to collect data about their owners in their normal environment in real time (ecological momentary assessment, EMA). This area of applications is becoming increasingly important for planning effective interventions because the data obtained in this way has greater credibility [22].

The widespread availability and use of smartphones is leading to greater access to mHealth applications than to the previous solutions of eHealth and telemedicine. In many countries, the number of smartphone users is growing rapidly and is equal to those using the stationary Internet. According to Newzoo report in 2018, smartphone penetration exceeds $50 \%$ in most European countries, from $53.8 \%$ in Romania, to $82.2 \%$ in the United Kingdom, and in Poland 64.0\% [23]. Forecasts show that the market size of mobile medical applications will grow from 2.4 billion USD in 2017 to 11.2 billion USD in 2025 [24].

The Research2Guidance report published in 2017 states that $325,000 \mathrm{mHealth}$ applications were available in the Google PlayStore store [25]. The estimated number of downloads of these applications by smartphone users was 3.7 billion in 2017. Clearly, the number of actual users of mobile applications is much lower, but enthusiastic business reports rarely indicate the potential user's disappointment with the installed software. This may also be confirmed by the same report stating that the supply of mHealth applications exceeds the demand. In addition, this sphere is characterised by high atomization as most applications are downloaded from online stores less than 5,000 times [25].

The development of the mHealth field is in harmony with the requirements proposed in relation to modern health care. The key strategies emphasise support for the independence of patients coping with a chronic disease. Mobile solutions are perfectly suited to the implementation of this strategy. A typical model of the mHealth system is founded on the possibility of access to health services based on Internet technologies without any restriction on time or place. As a result, different users can access health resources, such as electronic medical records, the systems to monitor symptoms and ailments, to facilitate managing a disease, or to support a healthy lifestyle and fighting addictions, without the limitations which exist in the traditional medical care and public health systems. For example, a doctor and other health care professionals can access the data generated by the patient virtually in real time. If the organisation of services allows it, the patient, in an emergency, can immediately ask for help from the doctor or his/her guardian in the system.

In 2011, the WHO published a compendium of the most promising new technologies, commercialised, or still at the development stage, which could meet the world's greatest health challenges [26]. Many of these technologies are mobile solutions, mostly based on the use of mobile telephony. The development of the applications highlighted by the WHO are for monitoring a patient's oxygen saturation of arterial blood, monitoring the activity of a foetus's heart, keeping health records of a child infected with HIV, transmitting images for diagnostic purposes and consultations with a doctor. According to the recent Research2Guidance report, the most attractive areas for companies developing mobile applications are communication with a doctor, diabetic and cardiological care, compliance with pharmacotherapy, health promotion and physical fitness, improvement of hospital performance and support for mental health [25].

\section{Areas of mHealth applications}

mHealth applications can be considered from the perspective of users, the available functionalities, medical specialties and the public health areas in which they are used. According to the authors of this paper, the key activities of mobile technologies in health care and public health include:

- monitoring of environmental conditions;

- monitoring of epidemiological phenomena;

- monitoring of disease development;

- $\quad$ supporting the treatment process;

- communicating between the health care worker and the patient, or between the health care professionals;

- promoting a healthy lifestyle;

- combating addiction.

The review of the previously referenced literature by Mechael and Sloninsky was intended to show the potential of the mHealth area and to systematise knowledge. According to the authors, the strongest trends in the development of mHealth applications are focused on areas like: emergency response, disease surveillance and control (e.g. malaria, HIV infection, tuberculosis, chronic diseases), coordination, management and supervision of human resources, mobile telemedicine in synchronous and asynchronous mode, support for decisions of clinicians in the place of care, remote monitoring and patient care, support to health including health promotion and community mobilization monitoring and reporting of health services, sharing m-learning resources regarding public health and supporting the continuous education of medical staff [1]. The same study included a list of the types of mobile technologies relevant for mHealth, spe- 
cifically mobile phones, personal digital assistants (PDA) and smartphones, devices for monitoring a patient's condition, mobile telemedicine devices providing telecare and mobile computing [1].

The Global Observatory for eHealth report, published in 2011, presented the results of a world survey of eHealth [27], which identified 6 activities implemented with the help of mHealth systems:

- communication between people and health care facilities (call centres with help lines);

- communication between the health care institution and a person or patient (compliance with therapeutic recommendations, reminders about appointments, mobilisation of the community, disseminating awareness of health issues);

- consultations between health care professionals (for the purposes of the survey, this type of communication was defined as mobile telemedicine);

- inter-sector communication in emergencies;

- health monitoring and supervision (mobile surveys, supervision, patient monitoring);

- access to information for health care workers in the place of care (information systems and decision support, patient documentation).

These activities differ from the results of earlier analyses based on scientific publications, which probably results from the rules governing the preparation of a survey addressed to the global community. The results of the survey show that the most common forms of health services implemented on the basis of mHealth solutions in the studied countries are call centres with help lines (59\%), free telephone services for emergency calls (55\%), emergency communication (54\%) and mobile telemedicine applications (49\%) [27].

For the purposes of a systematic review aimed at assessing the effectiveness of mHealth technology in improving health and health services, Free et al. proposed a division of mobile interventions focusing on a target group. According to this division, for the group of people carrying out research on health issues, mobile applications that collect data will be the main interest. In addition to these applications, healthcare professionals can also use solutions that support education and access to medical records. The applications used by both health care professionals and patients include those that enable the results of diagnostic tests to be sent, monitoring the course of a disease and supporting decision-making.

Applications designed mainly for patients are those that enable reminders of appointments to be sent, establishing a treatment plan, supporting the management of a chronic illness and increasing cooperation in taking prescribed medications. Patients and the members of a given population can use the applications that support behaviour modification, or deal with sudden health situations, such as those requiring first aid or posing a health threat. Mobile applications make it also possible to target the whole society with campaigns having health promotion objectives [28].

A systematic review carried out by Fiordelli et al. [29] considered the development of applications for mobile phones based on the analysis of publications describing the results of research on the impact of these devices on health outcomes. The authors focused on papers containing the results of testing mobile applications from 2002 to 2011. The number of publications meeting the review criteria increased from 1 to 30, of which the most popular, $63.2 \%$, were mobile applications designed for the need of care in cases of chronic diseases. $18.8 \%$ of applications supported preventive actions and the wellbeing of users and $17.9 \%$ of reports were applicable for acute situations. Of the papers covered by the review, the results of testing applications for patients with diabetes were found in $20.5 \%$, supporting the management of obesity and overweight in $13.7 \%$, mental health in $12.8 \%$, and helping to quit smoking in $8.5 \%$. The review also summarised the mobile functionalities employed. Text messages were used in $49 \%$ of the applications and ad hoc functions for specific diseases and health problems in $32 \%$. Devices that cooperate with mobile phones (referred to as "add-ons"), e.g. a glucometer for measuring glycemia or a pedometer for measuring physical activity, were used in $12 \%$ of the applications. Voice calls (10\%), video transmission (6\%) and multimedia messaging (3\%) were less frequently used. The authors also distinguished seven main areas of impact of the assessed mobile applications. The majority of applications were aimed at gaining the benefits of health promotion (38\%) and independence when dealing with a disease (33\%). Applications were used less frequently for communication improvement (22\%), remote monitoring (21\%), data collection $(21 \%)$, improved cooperation $(20 \%)$ and training and education (13\%).

The review prepared by Ali et al., which was published three years later, also attempted to evaluate the evolution and current state of research on mHealth, but less restrictive criteria were applied [30]. In addition, the review included articles published up to January 2015. As a result, the analysis covered as many as 515 publications. The authors distinguished three stages in the development of mHealth applications: the first, until 2006, in which PDA devices dominated; the second, from 2007 to 2012 , in which mainly the basic functions of mobile phones were employed and finally the period after 2012, in which smart devices dominated (smartphones, tablets with touch screens). Six main goals of mobile interventions were identified: health promotion, disease prevention, diagnostics, treatment, monitoring and support of health services. In the first stage of mHealth applications development, 51.5\% aimed at supporting health services and $33.3 \%$ focused on monitoring. None of the applications developed during this period was aimed at health promotion and disease prevention. In the second period, most applications were for monitoring (30.1\%) and health services support (25.9\%). Diagnostic applications accounted for $17.1 \%$, treatment applications for $16.1 \%$ and those for health promotion and disease prevention, only $10.9 \%$. The final stage of the development of the mHealth applications was characterised by the continued domination of the two most popular goal-oriented applications: those supporting health services, $25.3 \%$, moni- 
toring, 23.2\%. However, applications related to health promotion and disease prevention increased to $14.2 \%$. After 2012, in the last period of mHealth development, of the mobile applications relevant to disease states or health situations $57 \%$ related to non-communicable diseases and only $11.1 \%$ to infectious diseases, whilst those not related to a specific type of disease accounted for $14.5 \%$. Of the types of technologies used in this last stage of development of mHealth, $48.4 \%$ of applications were installed on a mobile device, $19.7 \%$ SMSs, $6.2 \%$ automatic sensors and $4.5 \%$ various forms of telephone conversation and video conferencing. In $9.7 \%$ of solutions, a mobile device was used to record, display, analyse and/ or transfer medical images and video material.

The most promising modern technologies include wearable devices. Health protection and public health are the key areas of their application, in addition to their use for communication, entertainment or business operations [31]. The wearable devices can be divided dependent on how they are worn [32]. For example, the wearables placed on the head include wireless headphones, sports or industrial helmets with headphones, a microphone, GPS location and display, contact lenses containing sensors for measuring glycemia, glasses enabling the display of information and application interfaces installed on the smartphone, implanted microphones referred to as "throat tattoo" which give voice commands, virtual reality helmets and augmented reality kits. One of the most popular places for wearing technology is the wrist or forearm. One can wear devices that look like a watch but are in fact portable voice activated computers, applications to monitor physical activity such as running, devices controlling other aspects of physical activity and devices for GPS location. Other examples include implantable subcutaneous RFID sensors with numerous applications such as door opening, shopping or accessing computers. Practically, wearable technology devices for specific applications can now be fitted to each part of the human body. Furthermore, swallowing devices, which have long been used in for gastroenterological diagnostics, could possibly be classified as wearable items.

\section{Assessment of the effectiveness of mhealth systems}

The results of secondary studies on the efficiency and suitability of mHealth applications in various areas are discussed below. As the number of papers on interventions with the use of mobile technologies in health care and public health, published in recent years, is very large, only the results of the most recent systematic reviews are presented. Since the following review of secondary research is selective, it does not enable definitive conclusions on the possibility of using mHealth solutions in specific fields to be made. The authors tried to show how the interest in mHealth in medical and public health environments has been raised, the size of the scope of mobile solutions and what kind of measures of effectiveness are used in such interventions. Because of the overwhelming number of publications of a synthetic character, the authors restricted themselves to presenting mainly the results of studies published in 2018. For selected areas, older publications, but presented in the last 5 years, were also considered.

\subsection{Health promotion and disease prevention}

A broader view of the trends in the development of mHealth solutions shows that health promotion is a particularly important area for their application. There is a huge number of mobile applications available in online shops that aim at propagating a healthy lifestyle, changing health behaviour and combating addictions. The search for publications that bring the synthesis of evidence leads to the conclusion that there is much research on interventions targeting lifestyle modification which make use of mobile technologies. This is evidenced by review studies assessing the effectiveness of such interventions being updated every year.

In 2015, a systematic review was published, in which an attempt was made to describe mobile applications used to modify behaviour [33]. The review included 24 studies mainly focusing on the assessment of usefulness and piloting small groups of participants. All the studies evaluated interventions that were developed on the basis of well-defined theories or strategies for changing behaviour and in 18 of the studies the intervention included self-monitoring. The interventions also included sending guidelines, feedback support for participants (9 studies each) and social support (7 studies). It is worth noting that the retention of participants, defined as a percentage of participants who remained in the study during the intervention and the observation period following the intervention, was $79.6 \%$ in the analysed research, but significantly different to individual studies which varied from $29.0 \%$ to $100.0 \%$. The effectiveness of mobile applications in achieving the intended goals was high. The effectiveness was confirmed in 8 out of 10 studies aimed at increasing physical activity. Of the 10 studies aimed at reducing the body mass index (BMI) or achieving weight reduction only one did not achieve the desired result. In three studies on depression, the symptoms of the illness were reduced. Two applications focused on combating alcohol addiction, one proved to be effective in reducing the number of days of risky drinking, and the other had no beneficial effect. In many studies, mobile applications were part of complex interventions rather than isolated activities.

In 2015, Hall et al. published a systematic review of reviews, which included 15 systematic reviews and meta-analyses published between 2009 and 2014, aimed at determining the effectiveness of interventions to improve health and change behaviour using text messaging (SMS) [34]. 228 studies on mHealth interventions aimed at modifying health behaviour concerning health promotion, disease prevention or self-management of chronic disease were analysed. It was confirmed that most of the interventions involving the transmission of text messages were effective in cases of the self-management of diabetes, weight loss, support for physical activity, the fight against smoking and for adherence to the pharmacother- 
apy recommended for AIDS. Unfortunately, the analysed interventions were characterised by high heterogeneity, which made it impossible to formulate recommendations with regard to the characteristics of the intervention. The long-term effects of such interventions are not known.

Dale et al. presented the results of a systematic review assessment of the effectiveness of mHealth interventions intended to modify a patient's behaviour related to lifestyle and compliance with a pharmacotherapy for treating cardiovascular disease [35]. Seven studies published by March 2015 were included in the review. In 5 out of 7 studies, it was demonstrated that mHealth interventions had beneficial effects by modifying behaviour. This was observed in the studies which evaluated the increase in physical activity and compliance with pharmacotherapy. However, no benefits were found for the interventions aimed at changing eating habits and alcohol consumption, or those aimed at the cessation of smoking. In four of the studies reviewed, clinical indicators were also evaluated, and in five studies - psychological indicators. In the first group, the beneficial effect of mHealth interventions involving the reduction of blood pressure was confirmed in two out of three studies. The interventions had no effect on blood lipids, BMI or on physical and functional fitness. In the second group of indicators, there was no reduction of anxiety and depressive symptoms, but in two of the three studies, the quality of life of the participants improved. The level of self-effectiveness increased only in one out of three studies of this characteristic.

In 2018, the results of a systematic review focusing on the effectiveness of mobile interventions made via a smartphone, which targeted the cessation of smoking, increased physical activity, a rational diet and reduction of alcohol consumption were published [36]. The analysis included 71 individual randomised controlled trials, of which 18 related to cessation of smoking, 44 to increased physical activity, improved diet or both, 2 to increased physical activity, improved diet and smoking cessation and 8 to the reduction of alcohol consumption. Mobile interventions used text message communication, telephone conversations, an interactive voice response system, applications installed on the phone, or a combination of these solutions. The collective analysis of the results showed that support by SMS increased the frequency of success in ceasing smoking. The review authors confirmed that mobile interventions aimed at improving the diet and increasing physical activity led to beneficial changes, although the magnitude varied. However, the assessment of the effects of interventions aimed at reducing alcohol consumption was not explicit.

The effectiveness of mobile applications in the field of modifying health behaviour was confirmed by Han and Lee [37]. Their review analysed 20 papers published between 2014 and 2017 giving the results of randomised controlled trials, related to a very wide range of health behaviour. The authors identified 12 areas: physical activity, alcohol consumption, smoking, diet, weight loss, compliance with medical recommendations, preparation for medical procedures, treatment of post-traumatic stress disorder, education during pregnancy, prevention of suicide, prevention of ischemic heart disease and knowledge about women's screening tests. In 16 of the 20 studies, the beneficial effects of mobile applications targeting health behaviour and clinical indicators were confirmed.

A small beneficial effect of interventions using mobile technologies to engender adherence to the principles of healthy eating was described by McCarroll et al. [38]. This was achieved in 5 of the 8 studies included in their analysis, in which nutritional habits were evaluated. In addition, 5 out of 13 studies showed a beneficial effect in terms of weight loss. Only randomised controlled trials published up to July 2016 were included in the review. Attention was drawn to the low quality of the research, which is a frequent situation in the case of a synthesis of the available evidence.

A meta-analysis, published by Cotie et al. assessed if eHealth interventions had an effect on the physical activity and obesity of women during their professional work [39]. The results of 20 out of 60 tests qualified for the review were included in the meta-analysis. The collective summary of the effect of eHealth interventions, many of which were implemented using mobile technologies, showed a statistically significant effect in increasing physical activity, but not on the indicators related to obesity (waist circumference, body weight, BMI).

The systematic review of Overdijkink et al. [40] gave an ambiguous assessment of the employment and effectiveness of mobile applications during pregnancy. The authors analysed 19 studies on shaping patients' lifestyle during pregnancy. In 14 of the 15 studies the usability of mobile applications was demonstrated. The interventions based on the use of mHealth solutions were effective in reducing excessive weight gain during pregnancy, increasing the consumption of fruit and vegetables and reducing smoking. The overview also provided the analysis of the results presented in 10 papers on the effectiveness of mobile interventions regarded as medical. They were focused on improving care in bronchial asthma, the treatment of diabetes and encouraging vaccination. In general, these studies did not show any benefits from the interventions, but an analysis undertaken on such a small number of individual studies would indicate that they were too small to reliably confirm any effectiveness.

The above review and that undertaken by Daly et al. [41] came from the journal 'JMIR mHealth and uHealth' and the systematic review of these authors had a similar goal. The aim was to determine the effect of mobile applications on health behaviour and health indicators in the perinatal period of pregnancy. It included only 4 randomised, controlled trials in which 456 women participated. In all studies, a relatively minor beneficial effect of mobile applications on primary indicators was demonstrated, including physical activity, the control of asthma symptoms, improvement of the stage of change (transition from a contemplation stage to a support stage according to the transtheoretical model), or the degree of the use of applications for seeking information about pregnancy and the level of patient activation. 


\subsection{Chronic diseases}

Improving care in chronic diseases has become a huge challenge for modern health care systems caused by the high financial and organisational demands associated with the services provided to patients with such conditions. It must be recognised that the occurrence of chronic diseases increases with age, and in many countries the increasingly aging population dominates. Therefore, there is a very significant interest in eHealth and mHealth systems that offer functions supporting patients' independence in coping with the disease; long-term monitoring of the disease based on independent measurements and observations; compliance with recommendations, especially in relation to pharmacotherapy; or easier communication with a doctor and other health care professionals. Selected studies on the effectiveness of interventions using mHealth solutions in many, or in single, chronic diseases are presented below.

In 2016, Whitehead and Seaton published the analysis of studies assessing the effectiveness of mobile applications installed on phones and tablets for improving the treatment of chronic diseases [42]. The review includes nine papers published in the period from 2008 to 2014 . All papers presented the results of randomised, controlled trials, in which mobile interventions were compared with standard care ( 8 studies) and, in one case, with an offline support program for self- management of a chronic disease. The chronic diseases considered were diabetes ( 5 papers), chronic lung diseases ( 3 papers) and a cardiovascular disease (1 paper). In all studies, the effectiveness of interventions was evaluated using indicators specific to the particular diseases. In 6 of the 9 studies, a significant impact of interventions on the primary indicators was found. Significant improvements in indicators typical for diabetes (HBA1c glycated hemoglobin) were found in four studies, two for type 1 diabetes and two for type 2 diabetes. In one study on patients with a cardiovascular disease, an improvement in the key clinical indicator was found (6-minute-walk test) in both subgroups subjected to active interventions. In the studies involving patients with respiratory diseases, a significant improvement in a respiratory function was demonstrated in one study on chronic obstructive pulmonary disease and in one of the two studies on bronchial asthma.

Beratarrechea et al. analysed the effects of interventions based on mHealth-related applications in chronic diseases in developing countries [43]. The analysis included 9 reports describing the results of randomised controlled trials. The results of the analysis confirmed the beneficial effect of mobile applications on measures related to the course of chronic diseases, visits to the doctor, clinical indicators and health-related quality of life. The cost effectiveness of this type of application was confirmed. Mobile technologies used in the interventions included text messaging, applications installed on smartphones and games, some of them associated with the use of wireless sensors. The authors of the review stated that strategies based on mHealth did not directly affect indicators related to obesity, such as BMI, but they led to in- creased physical activity, changes in diet and behavioural changes having an effect on body weight.

\section{Cardiovascular diseases}

Coorey et al. carried out a systematic review, in which the effectiveness, level of acceptance and usefulness of mobile applications to support patients' independent activities and self-management in cardiovascular diseases were evaluated [44]. The analysis included 10 studies with various methodologies such as open label randomised, controlled and uncontrolled trials, with the assessment before and after the intervention. These studies involved 607 patients with hypertension, heart failure, stroke or those participating in cardiological rehabilitation programmes. The beneficial impact of interventions based on the use of mobile technologies was based on the frequency of re-hospitalisation, quality of life, psychological well-being, blood pressure, BMI, waist circumference, blood cholesterol, exercise capacity, knowledge about the disease, compliance with pharmacotherapy and effectiveness of attempts to stop smoking. The key functionalities of mobile applications used in the studies included tracking health behaviour, self-monitoring of ailments, education about the disease and access to personalised content.

In 2018, the work of Liu et al. was published, in which the results of using mobile applications for the rehabilitation of patients suffering from ischemic heart disease were analysed [45]. 8 studies were included in their review which assessed the impact of mobile applications on the level of physical activity, adherence to the established treatment, the cessation of smoking, the level of anxiety and quality of life. According to the authors the results were inconsistent because of the significant limitations of individual tests, e.g. a small sample size, a too short observation period or too many patients withdrawing from the study. Out of 5 physical activity assessments, only two showed a beneficial effect. Two out of 4 studies confirmed the improvement of pharmacotherapy compliance, but only 1 of the 2 studies assessing smoking cessation showed any significant beneficial effect.

The usefulness of mobile applications to improve independence in the treatment of hypertension was analysed by Alessa et al. [46]. The authors reviewed 21 studies conducted on 3,112 patients. Of 14 studies aimed at reducing blood pressure, a significant benefit was confirmed in 10 cases: of which 6 were randomised controlled trials and 4 non-randomised trials. The authors of the review suggested that mobile applications that offer more functions may be more effective in reducing blood pressure. In virtually all cases, the study participants accepted the mobile applications and considered them as easy to use.

A systematic review published by Carbo et al. showed that the use of mHealth systems leads to beneficial effects related to the use of health care resources, costs and mortality in patients with heart failure [47]. In this review, 10 randomised controlled trials and one quasi-experimental study, involving 3,109 patients from North America 
and Europe, were analysed. Most of the study participants were monitored daily and controlled for an average of 6 months. Authors of the review found a significant reduction in the number of days spent in hospital due to heart failure. Statistically a non-significant reduction in expenses related to the disease, the number of hospitalisations, general mortality and disease-related mortality were also demonstrated. For various reasons there were no significant differences in the number and length of hospitalisations.

\section{Respiratory system diseases}

Most reports on the effectiveness of mobile applications in chronic obstructive pulmonary disease (COPD) and bronchial asthma related to their impact on the patients' greater independence and their ability to cope with the disease. High expectations were also associated with the use of eHealth systems in the care of patients with COPD. However, the latest systematic review prepared by Yang et al. may be disappointing [48]. Admittedly, the authors found that the trials they analysed may indicate a lower risk of hospitalisation in patients with COPD using applications installed on a mobile phone, but they failed to confirm the reduced periods of hospitalisation in the group using mobile solutions when compared to a group treated by standard methods. The systematic review included 8 studies in which interventions based on mobile applications enabling the recording and monitoring of physiological parameters (saturation of arterial blood oxygen, heart rate, blood pressure), physical activity (number of steps) and health behaviour (diet, the level of physical activity, taking medicines) were assessed. The assessment of the frequency and length of hospitalisation was based on the data from 6 trials for which the required information was available.

Miller et al. published a systematic review and a meta-analysis on the use of mobile technology interventions that support the independence of patients with bronchial asthma [49]. The review included 11 studies and the meta-analysis 9 studies. The results of the analysis revealed that mHealth interventions led to greater benefits in the field of pharmacotherapy and clinical effects compared to standard care. However, mobile interventions did not bring greater benefits than monitoring based on paper documentation of symptoms.

10 studies were appropriate for a systematic review prepared by Farzandipour et al. [50]. This review shows that mHealth applications led to better control of asthma symptoms (5 studies), lung function (2 studies) and quality of life ( 3 studies). There was no statistically significant increase in patients' self-efficacy (2 studies). The economic effects of the use of mobile applications were not unambiguous.

In 2018, a review was published regarding the suitability of applications installed on smartphones that encourage teenagers suffering from bronchial asthma to manage their own disease [51]. The analysis included 8 studies using very different methodologies. The trials included in the studies were very limited as they were restricted to 21 patients. Nevertheless, the authors emphasized the beneficial effect of interventions using mobile applications in respect of better control of bronchial asthma symptoms, compliance with therapeutic recommendations and greater self-efficacy.

The meta-analysis prepared by Xiao et al. evaluated the effectiveness of mHealth interventions in supporting the self-management of the disease in patients with bronchial asthma [52]. The meta-analysis of 18 studies showed that interventions with mHealth solutions led to better control of asthma symptoms, greater adherence to therapeutic recommendations, less frequent exacerbation of the disease and hospital admissions than with standard care.

\section{Diabetes}

In 2016, Hou et al. published the meta-analysis of 14 randomised clinical trials on the use of applications installed on mobile phones to improve the care of patients with diabetes [53]. 1,360 patients participated in the 14 studies. In all the studies in which patients with type 2 diabetes participated, a reduction in HbA1c occurred. The average reduction in its level in the patients using the mobile application was higher by $0.49 \%$ than in a control group. Greater benefits were found for younger patients and in cases where the use of the application was associated with the feedback information sent by health care professionals.

After analysing 20 studies on the use of mobile applications in type 2 diabetes Fu et al. concluded that despite reported significant problems with the usability of this type of solutions, they may lead to better glycemic control [54]. In the analysed studies, clinical efficacy was assessed on the basis of HbA1c measurements - a reduction in its level by $0.15 \%$ to $1.9 \%$ was observed. More optimistic results were obtained by Bonoto et al. [55]. The authors included 13 randomised, controlled trials in which 1,263 patients participated in their meta-analysis. 6 studies showed a statistically significant reduction in $\mathrm{HbA} 1 \mathrm{c}$ in the intervention group using a mobile application.

In 2017, an overview of systematic reviews focused on the assessment of the effectiveness of mHealth interventions in diabetes was published in the PLoS One magazine [56]. The authors, Kitsiou et al., analysed 15 systematic reviews of varying quality published between 2008 and 2014. They showed that interventions using mHealth solutions led to better control of glycemia compared to standard care or other interventions which did not make use of mHealth applications. The mean change in $\mathrm{HbA} 1 \mathrm{c}$ concentration in patients with type 2 diabetes was higher by $0.8 \%$ after using mHealth application and by $0.3 \%$ in patients with type 1 diabetes in the short-term perspective of 12 months.

In the same year, Wang et al. published a systematic review of the effectiveness of mHealth interventions in supporting independence and treatment of obesity and diabetes [57]. 24 papers from the years 2000-2016 were reviewed, in which the primary efficacy indices 
were weight loss or lowering the level of glycemia (evaluated on the basis of HbA1c). Interventions used in individual studies varied in length from 1 week to 24 months. According to the authors, more than half of the studies showed beneficial effects of interventions in relation to these primary indicators, but their limited size and quality did not enable credible recommendations to be made.

\section{Mental health}

The latest systematic review of the effectiveness of mobile applications in monitoring and treating symptoms of mental illnesses was published in 2018 by Wang et al. [58]. The authors selected 17 papers for the review, which described 16 mobile applications designed for people suffering from anxiety or stress, alcoholism, sleep disorders, depression, post-traumatic stress disorder (PTSD) and those exhibiting suicidal behaviour. Randomisation of participants for intervention was carried out only in 5 of the studies. In the opinion of the authors, a clinically significant reduction in the severity of symptoms or mental illness was demonstrated for 14 of the tested applications.

\section{Medical rehabilitation}

An important area where mHealth systems can be applied is rehabilitation. Physiotherapists may use a range of functionalities offered by mobile systems in their work [59]. These include monitoring the effects of exercise at home undertaken by the patient, collecting the measured reliable indicators and physiological parameters, correcting the posture and mechanics of body movements during exercises performed by the patient, providing educational materials and sending motivating messages to the patient. The importance of having access to intervention resources based on scientific evidence was stressed.

Multiple Sclerosis (MS) is an example of a condition that requires long-term support by a physiotherapist. In the meta-analysis of 11 randomised controlled trials conducted by Rintala et al., the authors assessed the effectiveness of telerehabilitation for patients with MS using tools such as the Internet, telephone, pedometer and video games requiring physical activity [60]. Although mobile solutions were not a priority of this study, it is worth emphasising that communication by telephone was an important element of the intervention. In 4 studies a mobile phone was used in conjunction with a pedometer. The cumulative result of the analysis indicated the significant beneficial effect of telerehabilitation on the physical activity of people suffering from MS.

\section{HIV}

A systematic review published in 2017 by Conserve et al. showed that text messages could induce people from a vulnerable population to undergo HIV testing [61]. The review was based on surveys carried out in countries with a medium to high national income.
The review of Amankwaa et al. showed that interventions based on text messaging led to an improvement in the use of anti-retroviral therapy in people infected with HIV [62]. Interactive voice response (IVR) did not have the same effect. The review was based on 13 studies and the accompanying meta-analysis was conducted on 11 .

\section{Limitations}

It must be emphasised that, despite the high expectations resulting from the use of mobile technologies in health care and public health, the potential benefits may be limited by at least several factors. The most important problems include the limited retention of mobile applications and wearable technology devices, the availability of a very large number of solutions of unknown value causing problems in selecting the most effective application and the lack of validation of the options offered by the health care or public health systems.

The key limitations identified by some authors are problems associated with the initial engagement and maintaining the involvement of the users of the mobile technologies to whom the interventions are addressed [63]. A significant percentage of the users of mobile health applications abandon them after only two weeks [64]. Similarly, 2 weeks is the period after which up to $50 \%$ of users part with wearable technology devices [65].

In response to some disappointment observed in the applications of mobile and wearable technologies in the area of health protection, health promotion and lifestyle support, some authors try to formulate principles of good practice in the design of such systems [66]. These recommendations include:

- limiting the scope of interaction required from the user;

- avoiding the enforcement of new behaviour and instead enabling the user to make adjustments on the basis of new information or the possibilities offered by the wearables;

- using the potential of networking by increasing the number of people using wearable technology devices, data bases, other devices, systems, services and software;

- avoiding the overload of the users with the volume of information; users may have problems using the information during short interactions with a wearable device;

- functionalities that send reminders, alarms and contextual information should be developed very carefully;

- the ability to communicate seems to be more important than the desire to display the data.

\section{Conclusions}

The evaluation of the suitability and effectiveness of mobile solutions is hampered by the lack of a uniform evaluation methodology. This is confirmed by the analysis carried out by McKay et al. in relation to mobile applications aimed at modifying health behaviour [67]. 
It is clear that mobile technologies are of great interest to health care and public health employees, patients and citizens. However, as in many areas subjected to a rigorous assessment, in the search of evidence justifying the application of interventions to improve health or cure diseases, there is some disappointment with the quality of the available evidence. Many studies are driven more by the enthusiasm of people fascinated by technological progress than a rational consideration of the benefits gained. The authors of this paper did not intend to carry out a systematic review of systematic reviews, but rather attempted to show what the results of the latest collective studies indicate about the possibilities of mHealth technologies. It is worth emphasising that for at least 3 years many systematic reviews and meta-analyses have been published each year, relating to the effectiveness of mHealth in various areas.

Currently, a mobile phone, usually a smartphone, is a personal device that allows the implementation of many functions essential for one's life and work. Its multi-functionality, combined with continuous access to the Internet and the possibility of adding additional peripheral devices, e.g. intelligent sensors, makes it an almost perfect tool at the current stage of technical development for supporting health-related behaviour and monitoring health conditions. The results of the reviews cited here are not always optimistic. In part, this is probably because the testing methods are imperfect and, partly because of the existing objective restrictions on the use of mobile technologies in the health context. In addition, much depends on the group targeted for mHealth interventions. However, it would appear that there is no turning away from mHealth developments and the reasonable scenario lies in the optimum use of its full potential, rather than questioning its significance.

\section{References}

1. Mechael P., Sloninsky D., Towards the development of an mHealth strategy: A literature review, World Health Organization, The Millenium Villages Project, 2008; http://www. who.int/goe/mobile_health/mHealthReview_Aug09.pdf(accessed: 5.11.2018).

2. Gernsback H. (ed.), Radio News Magazine [cover]. Experimenter Publishing Company, April 1924.

3. Gernsback H., The radio teledactyl, Science Invention, February 1925.

4. Wittson C.L., Benschoter R., Two-way television: helping the medical center reach out, "American Journal of Psychiatry" 1972; 129 (5): 624-627.

5. Bird K.T., Teleconsultation: a new health information exchange system, Massachusetts General Hospital, 1971.

6. Willemain T.R., Mark, R.G., Models of remote health care systems, "Biomedical Sciences Instrumentation" 1971; 8: 9-17.

7. Bashshur R.L., Armstrong P.A., Youssef Z.I., Telemedicine and medical care. Telemedicine: Explorations in the use of telecommunications in healthcare, Thomas, Springfield, USA 1975: 89-112.

8. Loman P., E-health: putting health on the Net, A First Consulting Group White Paper, 1999.
9. Oh H., Rizo C., Enkin M., Jadad A., What is eHealth (3): A systematic review of published definitions, "Journal of Medical Internet Research" 2005; 7(1): e1.

10. Eysenbach G., What is e-health?, "Journal of Medical Internet Research" 2001; 3 (2): e20.

11. World Health Organization Regional Office for Europe, E-health; http://www.euro.who.int/en/health-topics/Healthsystems/e-health (accessed: 15.11.2018).

12. Bashshur R., Shannon G., Krupinski E., Grigsby J., The taxonomy of telemedicine, "Telemedicine Journal and e-Health" 2011; 17 (6): 484-494.

13. Laxminarayan S., Istepanian R.S., UNWIRED E-MED: The next generation of wireless and internet telemedicine systems, "IEEE Transactions on Information Technology in Biomedicine" 2000; 4 (3): 189-193.

14. Dubendorf V.A., A history of wireless technologies, in: Dubendorf V.A. (ed.), Wireless Data Technologies, Jonh Wiley \& Sons, Ltd., ? 2003: 1-6.

15. Istepanian R.S., Lacal J.C., Emerging mobile communication technologies for health: some imperative notes on $m$-health, Engineering in Medicine and Biology Society, Proceedings of the $25^{\text {th }}$ Annual International Conference of the IEEE, 2003; 2: 1414-1416.

16. Istepanian R.S., Jovanov E., Zhang Y.T., Guest editorial introduction to the special section on m-health: Beyond seamless mobility and global wireless health-care connectivity, "IEEE Transactions on Information Technology in Biomedicine" 2004; 8 (4): 405-414.

17. Siau K., Shen Z., Mobile communications and mobile services, "International Journal of Mobile Communications" 2003; 1: 3-14.

18. Hayes J., XII things you (probably) didn't know... about watches, "Engineering \& Technology" 2013; 8 (12): 39-42.

19. Dunn J., Runge R., Snyder M., Wearables and the medical revolution, "Personalized medicine" 2018; 15 (5): 429-448.

20. TP-Link, ,Wearables” po polsku, Informacja prasowa 10 września 2015; http://prasa.tp-partner.pl/07-09_2015/ links/1.pdf (accessed: 17.11.2018).

21. Oberg P.A., Spelman F.A., Togawa T., Historical breakthroughs in medical sensing science, in: Oberg P.A., Togawa T., Spelman F.A. (eds.), Sensors in medicine and health care, John Wiley \& Sons, ? 2006: 3.

22. Shiffman S., Stone A.A., Hufford M.R., Ecological momentary assessment, "Annual Review of Clinical Psychology" 2008; 4: 1-32.

23. Newzoo, Top 50 countries/markets by smartphone users and penetration, September 2018; https:/newzoo.com/ insights/rankings/top-50-countries-by-smartphone-penetration-and-users/ (accessed: 17.11.2018).

24. Statista, The Statistics Portal, Mobile medical apps market size worldwide in 2017 and a forecast for 2025, Statista 2018; https://www.statista.com/statistics/877758/globalmobile-medical-apps-market-size/ (accessed: 21.11.2018).

25. Research2Guidance, mHealth App Economics 2017: current status and future trends in mobile health, 2017; https://www.uzelf.org/wp-content/uploads/2017/12/R2GmHealth-Developer-Economics-2017-Status-And-Trends. pdf (accessed: 17.11.2018).

26. World Health Organization, Compendium of new and emerging health technologies, 2011; http://apps.who.int/iris/bit- 
stream/handle/10665/95786/WHO_HSS_EHT_DIM_11.02 eng.pdf? sequence=1\&isAllowed=y (accessed: 15.11.2018).

27. World Health Organization, mHealth: New horizons for health through mobile technologies: second global survey on eHealth, 2011; https://www.who.int/goe/publications/ goe_mhealth_web.pdf (accessed: 16.11.2018).

28. Free C., Phillips G., Felix L., Galli L., Patel V., Edwards P., The effectiveness of M-health technologies for improving health and health services: a systematic review protocol, "BMC Research Notes" 2010; 3: 250.

29. Fiordelli M., Diviani N., Schulz P.J., Mapping mHealth research: a decade of evolution, "Journal of Medical Internet Research" 2013; 15 (5): e95.

30. Ali E.E., Chew L., Yap K.Y.L., Evolution and current status of mhealth research: A systematic review, "BMJ Innovations" 2016; 2 (1): 33-40.

31. Beecham Research Ltd., Wearable Technologies AG, Wearable Technology: Towards Function With Style, 2013; http:// www.beechamresearch.com/files/BRL\%20Wearable\%20 Tech\%20Report\%20Outline.pdf (accessed: 17.11.2018).

32. Raconteur, Wearable technology, 2015; https://raconteur. uberflip.com/i/565601-wearable-technology/10 (accessed: 15.11.2018).

33. Payne H.E., Lister C., West J.H., Bernhardt J.M., Behavioral functionality of mobile apps in health interventions: a systematic review of the literature, "JMIR mHealth and uHealth" 2015; 3 (1): e20.

34. Hall A.K., ColeLewis H., Bernhardt J.M., Mobile text messaging for health: a systematic review of reviews, "Annual Review of Public Health" 2015; 36: 393-415.

35. Dale L.P, Dobson R., Whittaker R., Maddison R., The effectiveness of mobile-health behaviour change interventions for cardiovascular disease self-management: A systematic review, "European Journal of Preventive Cardiology" 2016; 23 (8): 801-817.

36. Palmer M., Sutherland J., Barnard S., Wynne A., Rezel E., Doel A., GrigsbyDuffy L., Edwards S., Russel S., Hotopf E., Perel P., Free C., The effectiveness of smoking cessation, physical activity/diet and alcohol reduction interventions delivered by mobile phones for the prevention of noncommunicable diseases: A systematic review of randomised controlled trials, "PloS One" 2018; 13 (1): e0189801.

37. Han M., Lee E., Effectiveness of mobile health application use to improve health behavior changes: A systematic review of randomized controlled trials, "Healthcare Informatics Research" 2018; 24 (3): 207-226.

38. McCarroll R., Eyles H., Mhurchu C.N., Effectiveness of mobile health (mHealth) interventions for promoting healthy eating in adults: A systematic review, "Preventive Medicine" 2017; 105: 156-168.

39. Cotie L.M., Prince S.A., Elliott C.G., Ziss M.C., McDonnell L.A., Mullen K.A., Hiremath S., Pipe A.L., Reid R.D., Reed J.L., The effectiveness of eHealth interventions on physical activity and measures of obesity among workingage women: A systematic review and meta-analysis, "Obesity Reviews" 2018; 19 (10): 1340-1358.

40. Overdijkink S.B., Velu A.V., Rosman A.N., van Beukering M.D., Kok M., SteegersTheunissen R.P., The usability and effectiveness of mobile health technologybased lifestyle and medical intervention apps supporting health care dur- ing pregnancy: systematic review, "JMIR mHealth and uHealth" 2018; 6 (4): e109.

41. Daly L.M., Horey D., Middleton P.F., Boyle F.M., Flenady $\mathrm{V}$., The effect of mobile app interventions on influencing healthy maternal behavior and improving perinatal health outcomes: systematic review, "JMIR mHealth and uHealth" 2018; 6 (8): e10012.

42. Whitehead L., Seaton P., The effectiveness of self-management mobile phone and tablet apps in long-term condition management: A systematic review, "Journal of Medical Internet Research" 2016; 18 (5): e97.

43. Beratarrechea A., Lee A.G., Willner J.M., Jahangir E., Ciapponi A., Rubinstein A., The impact of mobile health interventions on chronic disease outcomes in developing countries: a systematic review, "Telemedicine Journal and e-Health" 2014; 20 (1): 75-82.

44. Coorey G.M., Neubeck L., Mulley J., Redfern J., Effectiveness, acceptability and usefulness of mobile applications for cardiovascular disease self-management: Systematic review with meta-synthesis of quantitative and qualitative data, "European Journal of Preventive Cardiology" 2018; 25 (5): 505-521.

45. Liu H., Xiao X., Lu C.M., Ling D.L., Wei R.H., A systematic review of the effect of mobile health on cardiac rehabilitation among coronary heart disease patients, "Frontiers of Nursing" 2018; 5 (3): 217-226.

46. Alessa T., Abdi S., Hawley M.S., de Witte L., Mobile apps to support the selfmanagement of hypertension: Systematic review of effectiveness, usability, and user satisfaction, "JMIR mHealth and uHealth" 2018; 6 (7): e10723.

47. Carbo A., Gupta M., Tamariz L., Palacio A., Levis S., Nemeth Z., Dang S., Mobile technologies for managing heart failure: A systematic review and metaanalysis, "Telemedicine and e-Health" (in print).

48. Yang F., Wang Y., Yang C., Hu H., Xiong Z., Mobile health applications in self-management of patients with chronic obstructive pulmonary disease: A systematic review and meta-analysis of their efficacy, "BMC Pulmonary Medicine" 2018; 18 (1): 147.

49. Miller L., Schüz B., Walters J., Walters E.H., Mobile technology interventions for asthma selfmanagement: systematic review and meta-analysis, "JMIR mHealth and uHealth" 2017; 5 (5): e57.

50. Farzandipour M., Nabovati E., Sharif R., Arani M.H., Anvari S., Patient selfmanagement of asthma using mobile health applications: A systematic review of the functionalities and effects, "Applied Clinical Informatics" 2017; 8 (4): 1068-1081.

51. Alquran A., Lambert K., Farouque A., Holland A., Davies J., Lampugnani E.R., Erbas B., Smartphone applications for encouraging asthma self-management in adolescents: A systematic review, "International Journal of Environmental Research and Public Health” 2018; 15 (11): 2403.

52. Xiao Q., Wang J., Chiang V., Choi T., Wang Y., Sun L., $\mathrm{Wu}$ Y., Effectiveness of mHealth interventions for asthma self-management: A systematic review and meta-analysis, "Studies in Health Technology and Informatics" 2018; 250: 144-145.

53. Hou C., Carter B., Hewitt J., Francisa T., Mayor S., Do mobile phone applications improve glycemic control (HbAlc) 
in the self-management of diabetes? A systematic review, meta-analysis, and GRADE of 14 randomized trials, "Diabetes Care" 2016; 39 (11): 2089-2095.

54. Fu H., McMahon S.K., Gross C.R., Adam T.J., Wyman J.F., Usability and clinical efficacy of diabetes mobile applications for adults with type 2 diabetes: A systematic review, "Diabetes Research and Clinical Practice" 2017; 131: 70-81.

55. Bonoto B.C., de Araújo V.E., Godói I.P., de Lemos L.L., Godman B., Bennie M., Diniz L.M., Junior A.A., Efficacy of mobile apps to support the care of patients with diabetes mellitus: A systematic review and metaanalysis of randomized controlled trials, "JMIR mHealth and uHealth" 2017; 5 (3): e4

56. Kitsiou S., Paré G., Jaana M., Gerber B., Effectiveness of mHealth interventions for patients with diabetes: an overview of systematic reviews, "PloS One" 2017; 12 (3): $\mathrm{e} 0173160$.

57. Wang Y., Xue H., Huang Y., Huang L., Zhang D., A systematic review of application and effectiveness of mHealth interventions for obesity and diabetes treatment and selfmanagement, "Advances in Nutrition" 2017; 8 (3): 449-462.

58. Wang K., Varma D.S., Prosperi M., A systematic review of the effectiveness of mobile apps for monitoring and management of mental health symptoms or disorders, "Journal of Psychiatric Research" 2018; 107: 73-78.

59. Dicianno B.E., Parmanto B., Fairman A.D., Crytzer T.M., Yu D.X., Pramana G., Coughenour D., Petrazzi A.A., Perspectives on the evolution of mobile (mHealth) technologies and application to rehabilitation, "Physical Therapy" 2015; 95 (3): 397-405.

60. Rintala A., Hakala S., Paltamaa J., Heinonen A., Karvanen J., Sjögren T., Effectiveness of technologybased distance physical rehabilitation interventions on physical activity and walking in multiple sclerosis: A systematic review and meta-analysis of randomized controlled trials, "Disability and Rehabilitation" 2018; 40 (4): 373-387.

61. Conserve D.F., Jennings L., Aguiar C., Shin G., Handler L., Maman S., Systematic review of mobile health behavioural interventions to improve uptake of HIV testing for vulnerable and key populations, "Journal of Telemedicine and Telecare" 2017; 23 (2): 347-359.

62. Amankwaa I., Boateng D., Quansah D.Y., Akuoko C.P., Evans C., Effectiveness of short message services and voice call interventions for antiretroviral therapy adherence and other outcomes: A systematic review and meta-analysis, "PloS One" 2018; 13 (9): e0204091.

63. Birnbaum F., Lewis D.M., Rosen R., Ranney M.L., Patient engagement and the design of digital health, "Academic Emergency Medicine” 2015; 22 (6): 754-756.

64. Appboy, Spring 2016 mobile customer retention report: an analysis of retention by day, Braze, New York (NY) 2016.

65. Shih P.C., Han K., Poole E.S., Rosson M.B., Carroll J.M., Use and adoption challenges of wearable activity trackers, I Conference 2015 Proceeding 2015 Mar 24-27, iSchools, Newport Beach, CA, 2015; https://www.ideals. illinois.edu/bitstream/handle/2142/73649/164_ready. pdf?sequence=2\&isAllowed=y (accessed: 18.11.2018).

66. Khan S., Marzec E., Wearables. On-body computing devices are ready for business, in: Briggs B., Tech Trends 2014 Inspiring Disruption, Deloitte University Press 2014; https://www2.deloitte.com/content/dam/Deloitte/global/ Documents/Technology/gx-cons-tech-trends-2014-inspiring-disruption.pdf (accessed: 18.11.2018).

67. McKay F.H., Cheng C., Wright A., Shill J., Stephens H., Uccellini M., Evaluating mobile phone applications for health behaviour change: A systematic review, "Journal of Telemedicine and Telecare" 2018; 24 (1): 22-30. 